\title{
Colaboración interinstitucional: Reto y alternativa en la educación tecnológica
}

\author{
Edgar Alberto Mendoza Parada \\ Profesor Departamento de Tecnología \\ Universidad Pedagógica Nacional
}

\section{ANTECEDENTES}

Con el propósito fundamental de mejorar la calidad y la eficiencia de las Áreas Técnicas del aparato educativo estatal, en función del mercado laboral y de la satisfacción de necesidades regionales, el Gobierno Nacional ha impulsado desde 1985 la coordinación y complementación de los dos sistemas de educación: el de "Formal" a cargo del Ministerio de Educación y el de "capacitación y profesionalización" a cargo del SENA.

El punto de partida de este proceso de integración puede ubicarse en la Ley 55 de 1985, reorientadora de las finanzas del Estado, que entre otras disposiciones asigna al SENA la financiación total o parcial de los Institutos Técnicos y Escuelas Vocacionales. Directrices posteriores más particulares del Consejo Nacional de Política Económica y Social -CONIPES- (Enero 88 -Junio 88) establecen una clara diferenciación de competencias en el manejo de la Educación Media, asignando al sistema de educación formal (MEN) el desarrollo de las actitudes y conocimientos relacionados con los sabores universales, y al sistema de capacitación y formación profesional (SENA), el desarrollo de los conocimientos, habilidades y destrezas relacionados con la actividad laboral. Además, dispone que el SENA debe asumir la responsabilidad de orientación y atención de la componente técnica de la educación vocacional, al tiempo que establecer, en coordinación con el MEN, una política nacional de educación técnica y vocacional.

El mecanismo operativo mediante el cual se le dió cuerpo a la norma es el Convenio SENA-MEN, que tiene como objetivo principal, en su etapa actual de implementación, el diseño y desarrollo de una política nacional de educación técnica y, como actividad central, la articulación e integración de servicios a través de Proyectos Piloto Regionales. El esquema general de estos proyectos contempla estudios de oferta, demanda, diseño y adecuación curricular integrada, capacitación de docentes, diseño y reproducción de material didáctico, dotación y mantenimiento de equipos, apoyo a proyectos de formaciónproducción y construcción y mantenimiento de instalaciones locativas. Los proyectos se desarrollan en planteles oficiales con capacidad para que la experiencia pueda ser demostrativa a corto plazo y buscan cubrimiento nacional para que en conjunto puedan ser suficientemente representativas de las necesidades regionales y de diversas modalidades tecnológicas.

El Convenio opera bajo la dirección de un Comité Nacional en el que además de las instituciones titulares tiene participación el Departamento Nacional de Planeación y Comités Regionales integrados por los FER, Secretarías de Educación y SENA regionales. Su ejecución es realizada bajo la orientación de grupos técnicos constituidos por especialistas de las dos instituciones en las diferentes áreas de acción.

En sus cuatro años de vigencia, desde su primera formulación en Julio del 85 hasta la fecha, el Convenio ha sido nutrido por la creciente sensibilidad nacional de años recientes frente a la necesidad de construcción de un SISTEMA científico tecnológico nacional y ha 
evolucionado en sus objetivos y acciones, desde un énfasis en transferencia de recursos financieros del SENA al MEN, hacia la búsqueda de una reorientación de la educación técnica. Así mismo, la implementación del proceso de articulación, ha propiciado una muy positiva atmósfera de colaboración interinstitucional ante la magnitud, impacto y cobertura de los cambios que se están operando y que constituyen a la vez que un reto para todo el sistema, una ocasión única de confluencia de circunstancias que han permitido la constitución de un gran laboratorio educativo.

A través del largo proceso que ha de recorrerse desde las definiciones políticas hasta la implementación y evaluación de respuestas, la Universidad Pedagógica Nacional, por la naturaleza de sus obligaciones institucionales, podría aportar significativas contribuciones al tiempo que nutrir y enriquecer sus procesos internos.

El marco en el que tal contribución puede hacerse posible es, por una parte, el Convenio SENA-MEN que explícitamente contempla la posibilidad de "vinculación de otros organismos públicos y privados" y por la otra, el Convenio de complementación de servicios entre la UPN y el SENA, actualmente vigente. Son objetivos de este último, entre otros, "generar y definir tecnologías educativas para el desarrollo del País y realizar investigaciones, trabajos y prácticas docentes conjuntas», y la "realización de acciones conjuntas para la formación de personal de los niveles medio y superior» bajo el criterio de la optimización de recursos humanos, materiales y tecnológicos.

Si bien estos objetivos son de amplia cobertura y permiten, atendiendo las propias competencias institucionales, la exploración de una rica gama de proyectos que pudieran contribuir al mejoramiento de una misma función social dentro del campo de la educación técnica y tecnológica, las acciones emprendidas hasta ahora han sido de un alcance restringido en relación con las propias posibilidades del convenio y más aún, frente a las tendencias actuales de complementariedad entre los distintos organismos que participarían de lo que sería un gran sistema científico tecnológico nacional.

Se trataría ahora, con ocasión de la necesaria redefinición que habrá de producirse dentro de la educación básica y media, de imprimirle un mayor dinamismo al convenio vigente, integrando sus acciones en un programa coherente que en conjunto apoye las "Aseas de atención" definidas en las «nuevas orientaciones» del Convenio SENA - MEN.

Las acciones que hasta ahora se han desarrollado durante la vigencia del Convenio U7PN - SENA, han permitido mantener la relación interinstitucional y han demostrado en la práctica la posibilidad de éxito de trabajos conjuntos. Además de los cursos ofrecidos por el SENA como complemento a la formación de los estudiantes de la Facultad de Ciencia y Tecnología de la Universidad Pedagógica Nacional, destacable el Seminario que sobre Ciencia, tecnología y Sociedad fuera promovido por la misma Universidad, al que asistieran además de funcionarios del SENA, miembros de distintas dependencias del Ministerio de Educación Nacional.

Esta experiencia reprogramada conjuntamente podría ser extendida a una población más amplia y además integrarse a un programa de capacitación de doble vía entre las dos instituciones.

El trabajo recientemente coordinado por el SENA permitió identificar posibles proyectos interinstitucionales que además de posibilitar acciones a corto plazo, pueden constituirse en fuentes de validación para la tarea fundamental, cual es la contribución al establecimiento de una política nacional de educación técnica. Se trata básicamente de 
dos programas Mejoramiento dela calidad Docente y Divulgación Tecnológica, sobre los que se esbozan abajo algunas aproximaciones. Estos programas pueden basarse en un acuerdo mínimo sobre la importancia de concebir la educación tecnológica en los niveles básicos y medios, con un horizonte más amplio que el tradicional de capacitación laboral parcial o terminal-, con el que preferencialmente ha sido considerada hasta ahora y que incluyan explícitamente su contenido cultural y pedagógico. Así, serían tres las «razones» básicas que responderían a la pregunta de para qué la educación tecnológica en los mencionados niveles.

-Cultural Si se acepta que la Ciencia y la Tecnología se han constituido en parte integral de la cultura contemporánea, entonces la educación científica y tecnológica dirigida a los más amplios sectores de la población es un requisito de formación integral y un elemento básico para la comprensión del mundo contemporáneo.

- Pedagógico Se reconoce que la explicación tecnológica al integrar saber y acción y al requerir la confluencia de discursos explicativos de varias disciplinas, en función de la comprensión de objetos o situaciones tecnológicas, permite mejorar procesos y resultados de aprendizaje en otras áreas del conocimiento y establecer relaciones relevantes entre saberes particulares.

- Laboral Se trata de la formación en una rama específica del saber tecnológico, que conduzca directamente a una salida ocupacional o que se constituya en un eslabón dentro de un sistema articulado de educación tecnológica.

\section{PROGRAMA DE MEJORAMIENTO DE LA CALIDAD DOCENTE}

Un programa de profesionalización que conduzca a la obtención de un título académico en docencia en áreas tecnológicas, puede desarrollarse como respuesta a las necesidades detectadas de mejoramiento de la calidad profesional y condiciones laborales de un significativo sector de docentes que actualmente laboran en instituciones del sector educativo con una escolaridad no universitaria. Las experiencias educacionales del SENA y de la Universidad Pedagógica, unidas a la identificación de necesidades por parte del Ministerio de Educación Nacional y a las generadas dentro del convenio MENSENA a partir de los proyectos pilotos, podrían confluir en la elaboración de un programa académico conjunto, que puede concebirse de muy diversas maneras según sean las especificidades regionales, modalidades de trabajo académico: escolarizado o semidesescolarizado, etc.

Una posible estrategia es la ejecución de una experiencia piloto cerrada a una promoción y dirigida a pequeños grupos que, conformados según algún criterio de homogeneidad (área tecnológica, experiencia laboral, pertinencia a un programa regional, etc.), permita programar un currículo flexible que, conservando estándares de calidad, atienda tanto a la experiencia tecnológica y docente de los integrantes como a las limitaciones de tiempo que impone su ejercicio laboral.

Operativamente el programa sería diseñado conjuntamente entre el SENA y la Universidad Pedagógica Nacional buscando complementación de recursos y experiencias institucionales y estaría dirigido a instructores SENA y/o docentes MEN en grupos separados, o integrados para los casos en que las exigencias de las políticas definidas por el Convenio SENA-MEN lo demanden. 
Adicionalmente, pueden concebirse muchos y muy variados niveles de integración entre la UPN y el SENA como también varios "subprogramas" con relativa autonomía como tales, pero articulados en un programa macro de formación de docentes, en forma tal, que se conjuguen los objetivos de los actuales programas de Licenciaturas o de los nuevos que los resultados de la investigación generada por el Convenio SENA-MIEN sugieran crear, de acuerdo con las necesidades más urgentes de comunidades docentes particulares.

\section{DIVULGACION TECNOLOGICA}

Los riesgos de la divulgación, entendida como adaptación de conocimientos a la comprensión de públicos amplios no especializados, ha sido contundentemente expresada por Sábato: "No hay nada más peligroso que un poco de cultura". Esto, tomado como advertencia puede ser interpretado por lo menos en dos aspectos: uno, la pérdida de riqueza, profundidad y rigor del concepto transmitido, que es consecuencia de la adaptación de lenguajes e instrumentos lógicos, y otro, las implicaciones derivadas del manejo de conclusiones o resultados sin el conocimiento del proceso previo que las origina o sustenta, o del contexto teórico que les pueda definir sus límites de validez.

La divulgación de su misma naturaleza no puede aspirar a la difusión estricta y completa de contenidos. Pero esta limitación esencial no puede desvirtuar su utilidad social, que consiste en el sentido más amplio del término, en disponer a los hombres a la comprensión de la altura de los tiempos que les correspondió vivir. En el ámbito de la educación formal cabe preguntarse si la acción educativa en los niveles básicos, medios y buena parte de los universitarios, no es una actividad esencialmente divulgativa.

La falta de rigor en la transmisión y difusión del conocimiento es un riesgo propio ala actividad docente en cualquier nivel y un motivo de reflexión profundamente pedagógico. Del agente que lidera el proceso de adaptación de un conocimiento más elaborado y preciso, a otro que lo es menos, se espera que lo haga con el mayor sentido de rigor sobre la base del conocimiento más profundo y abstracto que se supone posee en relación con aquel que transmite. La producción de material de divulgación científico y tecnológico es una tarea que requiere de equipos profesionales de alta competencia, tanto en el manejo de medios de comunicación, como de dominio de las disciplinas particulares. La conformación de equipos que reúnan esas características o los esfuerzos por prepararlos parecen requerir un mayor apoyo. Así esbozada, la divulgación es un tema de la mayor pertinencia a los objetivos institucionales de la Universidad Pedagógica Nacional.

En un contexto de educación no formal, la divulgación se ha entendido de una manera más precisa y operativa y se refiere fundamentalmente a una labor que en sentido amplio puede considerarse como extensión comunitaria. Concebida en el SENA, como una "modalidad de acción a través de la cual, y sin la existencia del alumno como tal, la entidad pone al alcance de personas, empresas o unidades productivas, la información tecnológica que posee", se ha acumulado ya una amplia experiencia que, asimilada y enriquecida con recursos de otras instituciones, podría constituirse en un propósito nacional extendible a todos los niveles educacionales.

La Universidad Pedagógica Nacional podría contribuir a la definición de una política de divulgación y al estudio de sus aspectos intrínsecamente pedagógicos, ala elaboración de materiales, o a apoyar acciones que ya están en ejecución en el SENA. Una posible vía es la vinculación de grupos estudiantiles y profesorales a través de espacios académicos 
ya conformados como las prácticas docentes, monografías de pregrado y tesis de posgrado. La articulación de las labores académicas naturales a un institución universitaria a programas sistemáticos de incidencia nacional como los esbozados, le daría no solo mayor coherencia a estas actividades tan a menudo aisladas, sino también mayor utilidad social y representan, sin duda, una mejor utilización del recurso humano.

\section{BIBLIOGRAFIA}

SENA/MEN. Nuevas orientaciones del Convenio MEN-SENA. 1989-1990. Marco de referencia para la Programación Regional. Bogotá, Junio de 1989.

El Proceso Curricular Articulado de los Proyectos Piloto Regionales - Marco de referencia y orientaciones. Bogotá, Noviembre de 1989.

Evaluación Diagnóstica de la Educación Técnica. 1990. 